\title{
MORFOLOGIA COMPARADA DA CABEÇA DAS ESPÉCIES SULBRASILEIRAS DE MORPHINAE (LEPIDOPTERA, NYMPHALIDAE) ${ }^{1}$
}

\author{
Ivana Gavassi Bilotta ${ }^{2}$
}

\begin{abstract}
A COMPARED MORPHOLOGY OF THE HEAD OF THE SOUTH BRAZILIAN SPECIES OF MORPHINAE (LEPIDOPTERA, NYMPHALIDAE). This research presents a detailed study of the morphology of the head of adult in both sexes of the south Brazilian species of the Morphinae subfamily (Lepidoptera, Nymphalidae), as mentioned below: Morpho helenor violaceus Fruhstorfer, 1912; Iphimedeia hercules (Dalman, 1823); Iphixibia anaxibia (Esper, 1801); Cytheritis portis thamyris (Felder \& Felder, 1867); Cytheritis aega (Huebner, 1822); Pessonia catenaria (Perry, 1811); Grasseia menelaus nestira (Huebner, 1821).

KEY WORDS: Nymphalidae, Morphinae, morphology, head
\end{abstract}

Com mais de 5000 espécies, a família Nymphalidae é uma das mais numerosas entre os lepidópteros diurnos. Entre estas, está a subfamília Morphinae, distribuída na região Neotropical, desde o México até o norte da Argentina e Sul do Brasil. Dos oito gêneros aceitos para a subfamília, seis são tratados neste trabalho.

Devido aos poucos estudos relacionados com a organização das subfamílias de Nymphalidae, o presente trabalho tem como finalidade um levantamento pormenorizado dos dados morfológicos e zoogeográficos dos adultos das espécies sulbrasileiras de Morphinae. Este é a primeira parte de uma série de três trabalhos, onde está sendo comparada a morfologia externa da cabeça dos adultos.

\section{MATERIAL E MÉTODOS}

Os trabalhos de CASAGRANDE (1979); COSTA LIMA (1950); DOUBLEDAY (1846-1852); EATON (1971); EHRLICH (1958 A,B); MADDEN (1944); MATSUDA (1965); NICULESCU (1968, 1976, 1978); REUTER (1896, 1898); SBORDONI \& FORESTIERO (1985); SHORT (1951) E (SNODGRASS, 1935); foram de grande importância para o estudo morfológico comparativo interespecífico. Para o embasamento sistemático, os trabalhos utilizados foram: FRUHSTORFER (1912-1924); HARVEY (1991); LE MOULT \& RÉAL (1962-1963); STAUDINGER (1884-1888).

1) Contribuição número 782 do Departamento de Zoologia, Universidade Federal do Paraná.

2) Departamento de Zoologia, Universidade Federal do Paraná, Caixa Postal 19020, 81531-970 Curitiba, Paraná, Brasil. Boissista do CNPq. 
O material utilizado para o estudo, encontra-se depositado na Coleção de Entomologia Pe. J.S. Moure do Departamento de Zoologia, Setor de Ciências Biológicas da Universidade Federal do Paraná (UFPR).

Para o estudo da morfologia externa, a cabeça com seus apêndices foi destacada do restante do corpo; fervida numa solução de hidróxido de potássio (KOH 10\%), em banho maria durante alguns minutos, até seu amolecimento, facilitando assim a remoção dos músculos e das escamas e pêlos.

Foram examinados machos e fêmeas das seguintes espécies:

Morpho helenor violaceus Fruhstorfer, 1912; Iphimedeia hercules (Dalman, 1823); Iphixibia anaxibia (Esper, 1801); Cytheritis portis thamyris (Felder \& Felder, 1867); Cytheritis aega (Huebner, 1822); Pessonia catenaria (Perry, 1811); Grasseia menelaus nestira (Huebner, 1821).

Também foram examinadas duas subespécies e uma espécie próximas: Morpho helenor paulista Fruhstorfer, 1907; Morpho helenor achillaena (Huebner, 1823); Pessonia epistrophus (Fabricius, 1796) $(=$ P. laertes (Drury,1782)); mas como era de se esperar, não mostraram em sua estrutura morfológica diferenças significativas com outras da mesma espécie ou grupo; desta forma, não foram incluídas.

As estruturas da cabeça e seus apêndices são descritas e comparadas interespecificamente.

\section{CABEÇA}

Em todas as espécies estudadas, apresenta-se aproximadamente duas vezes mais larga que longa, proporcional ao corpo e sem ocelos (Figs. 1 a 28 ).

Os olhos compostos são grandes, proeminentes e glabros. A sutura ocular delimita uma área proximal aos olhos formando uma faixa estreita denominada esclerito ocular (Figs 1 a 28).

O frontoclípeo, de forma quadrangular, situado entre os olhos, está separado do labro através da sutura clipeolabral, dos alvéolos antenais pela sutura transfrontal e lateralmente pela sutura ocular (Figs 1 a 7 ).

Entre a sutura ocular e a sutura laterofacial encontra-se a área parocular, estreita e que termina logo acima da fóvea tentorial anterior, na região lateral e mediana do frontoclípeo (Figs. 1 a 7).

A fóvea tentorial anterior, na sutura subgenal; segundo SNODGRASS (1935), é marcada externamente por uma invaginação do exoesqueleto (Figs 1 a 7).

A sutura subgenal, continuação da sutura laterofacial, vai desde a fóvea tentorial anterior até a sutura clipeolabral. As suturas subgenal e ocular delimitam uma área triangular denominada área subgenal (Figs 1 a 7). a 14).

Os alvéólos antenais não se tocam na regiấo mediana da cabeça (Figs 8

O vértice, região dorsal da cabeça, é delimitado pela sutura transfrontal, lateralmente pelos alvéolos antenais e suturas temporais que se estendem até a sutura pós-ocipital (Figs 1 a 21). As espécies $P$. catenaria e G. menelaus nestira 

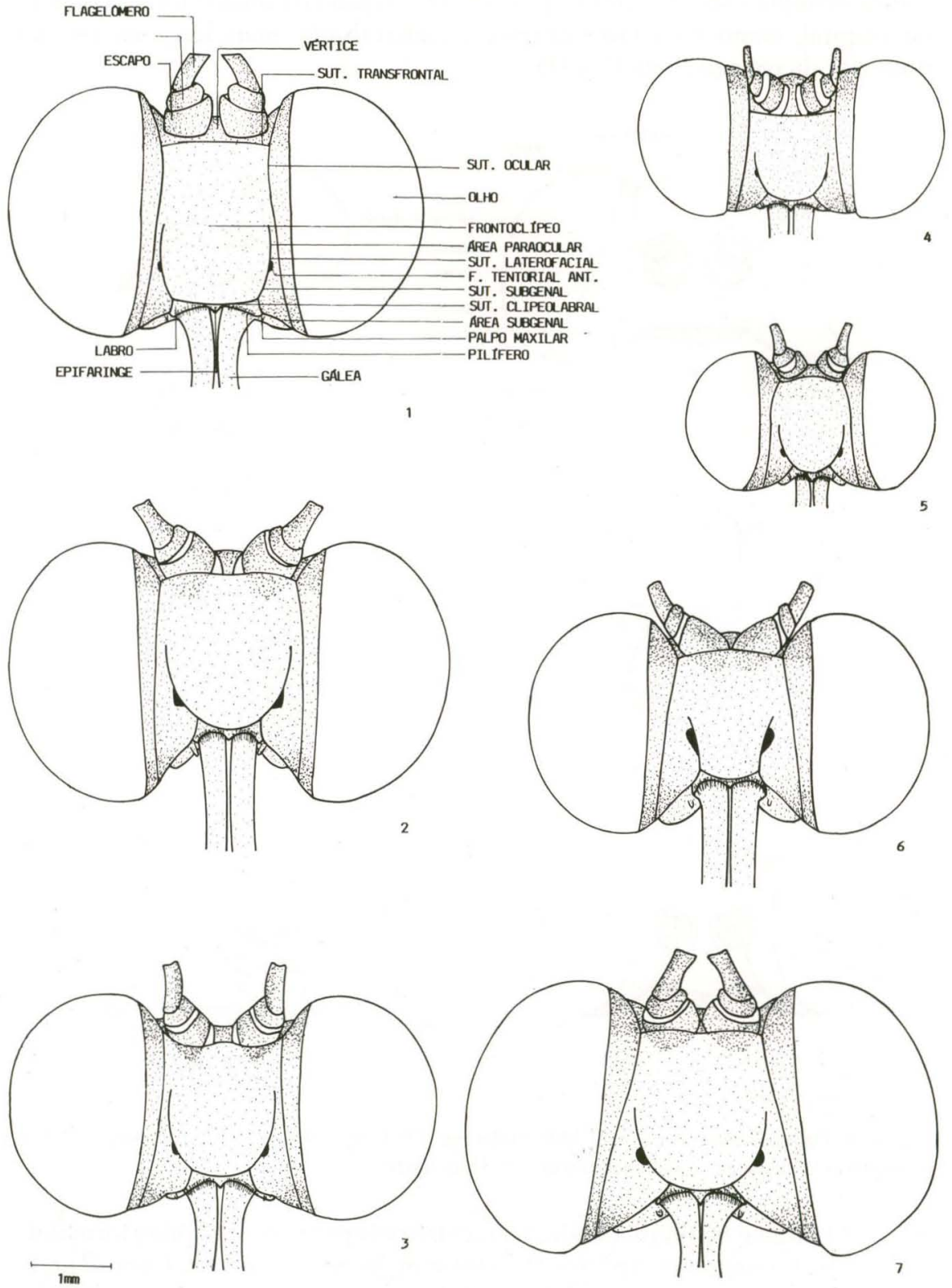

Figs 1-7. Cabeça: vista frontal. (1) M.h. violaceus; (2) I. hercules; (3) I. anaxibia; (4) C.p. thamyris; (5) C. aega; (6) P. catenaria; (7) G.m. nestira. 
apresentam uma área rugosa no vértice (Figs 13 e 14).

O ocipício localiza-se logo após ao vértice, até a sutura pós-ocipital. Esta sutura delimita todo o esclerito pós-ocipital, o qual circunda o forame magno ou ocipital, como uma faixa estreita e esclerotisada, mais larga na porção posterior da cabeça (Figs 15 a 21).
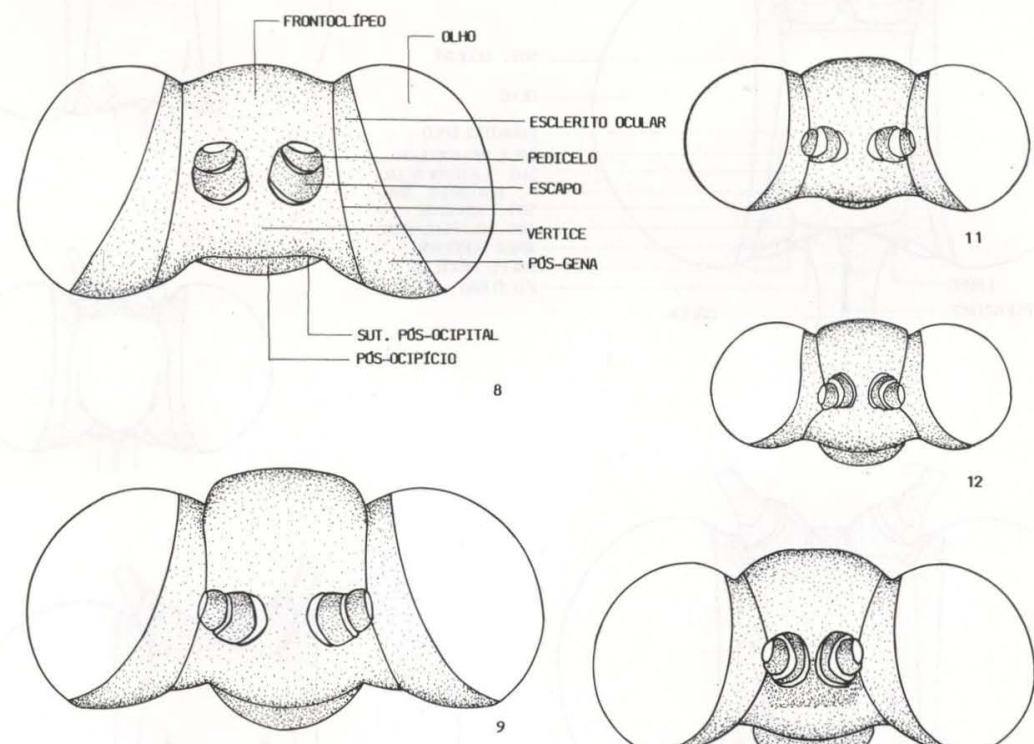

12

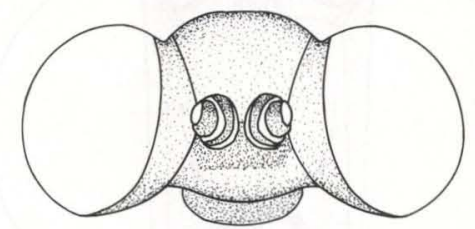

13

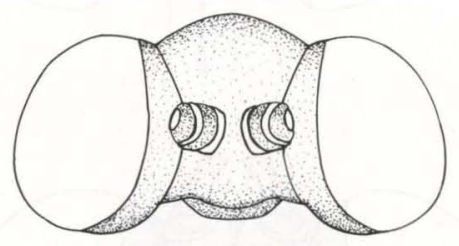

10

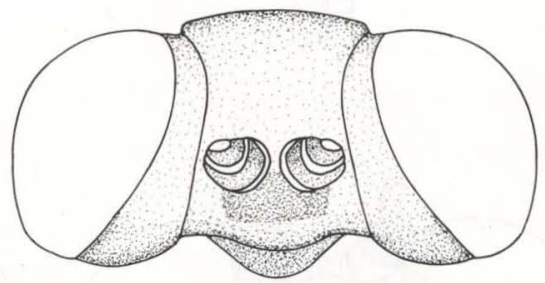

14

Figs 8-14. Cabeça: vista dorsal. (8) M.h. violaceus; (9) I. hercules; (10) I. anaxibia; (11) C.p. thamyris; (12) C. aega; (13) P. catenaria; (14) G.m. nestira.

O forame ocipital é dividido ao centro pela ponte pós-ocipital, formando assim dois forames. As espécies $M$. helenor violaceus, I. hercules, I. anaxibia, $C$. aega e G. menelaus nestira apresentam o forame superior maior que o inferior (Figs 15 a 17, 19 e 21); e C. portis thamyris e P. catenaria o superior e inferior são aproximadamente do mesmo tamanho (Figs 18 e 20). Quanto a forma, $I$. 

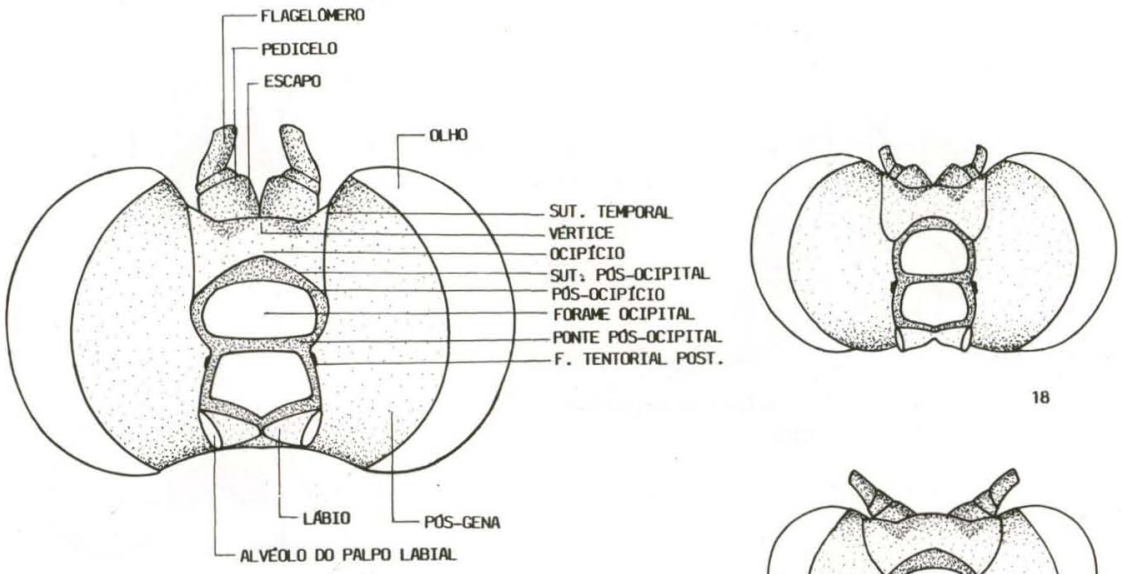

18
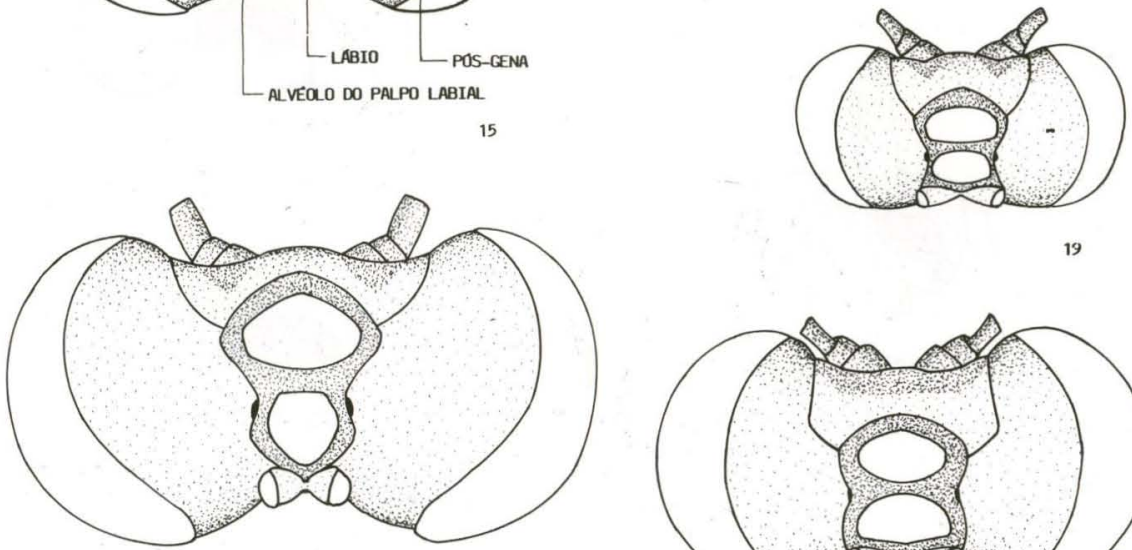

16

19

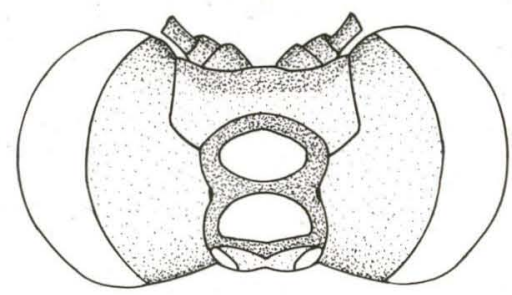

20
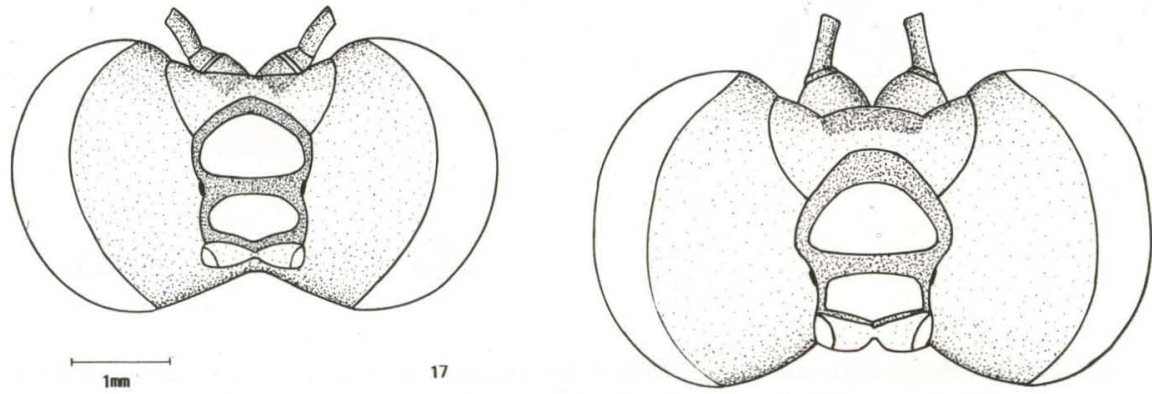

21

Figs 15 a 21. Cabeça: vista posterior. (15) M.h. violaceus; (16) I. hercules; (17) I. anaxibia; (18) C.p. thamyris; (19) C. aega; (20) P. catenaria; (21) G.m. nestira.

hercules, $I$. anaxibia, $C$. portis thamyris e $G$. menelaus nestira apresentam os forames superior e inferior triangulares (Figs 16 a 18 e 21); em $M$. helenor violaceus, o superior é oval e o inferior triangular (Fig. 15); em C. aega o superior é triangular e o inferior oval (Fig. 19) e em P. catenaria tanto o superior como o 

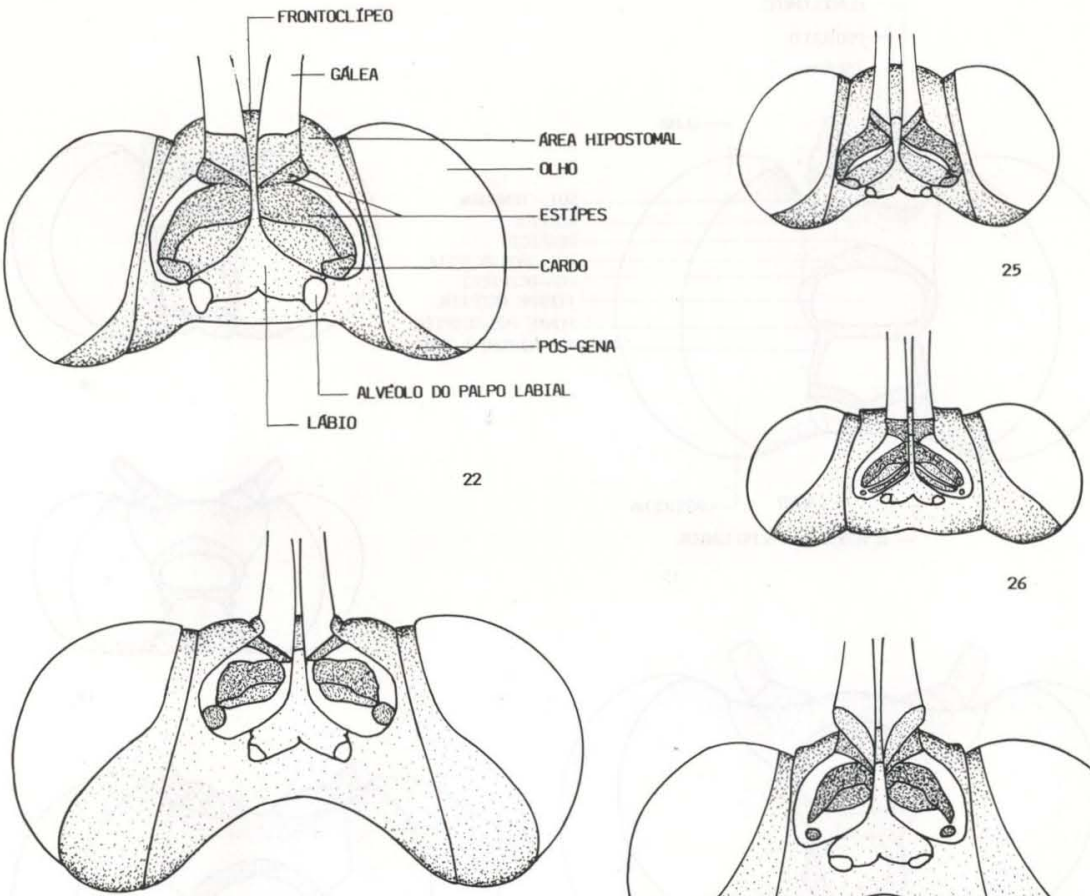

23
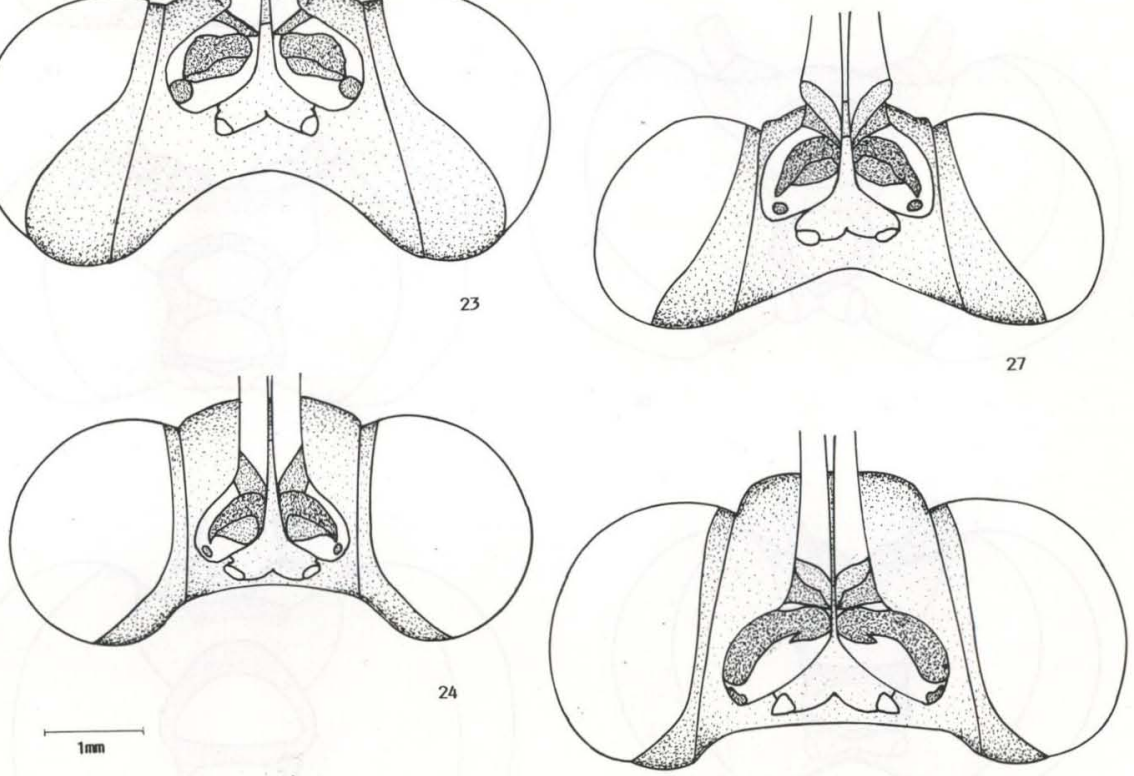

28

Figs 22 a 28. Cabeça: vista ventral. (22) M.h. violaceus; (23) I. hercules; (24) I. anaxibia; (25) C.p. thamyris; (26) C. aega; (27) P. catenaria; (28) G.m. nestira.

inferior tem o formato de meia esfera (Fig. 20). Localizada lateralmente à ponte pós-ocipital e ao lado da sutura pós-ocipital, está a fóvea tentorial posterior (Figs 15 a 21).

Ocupando toda região posterior, entre os olhos e o pós-ocipício, está a pós-gena estendendo-se ventralmente até a região sub-genal (Figs 15 a 21).

Ventralmente, a fossa proboscidial, que forma uma grande depressão, 

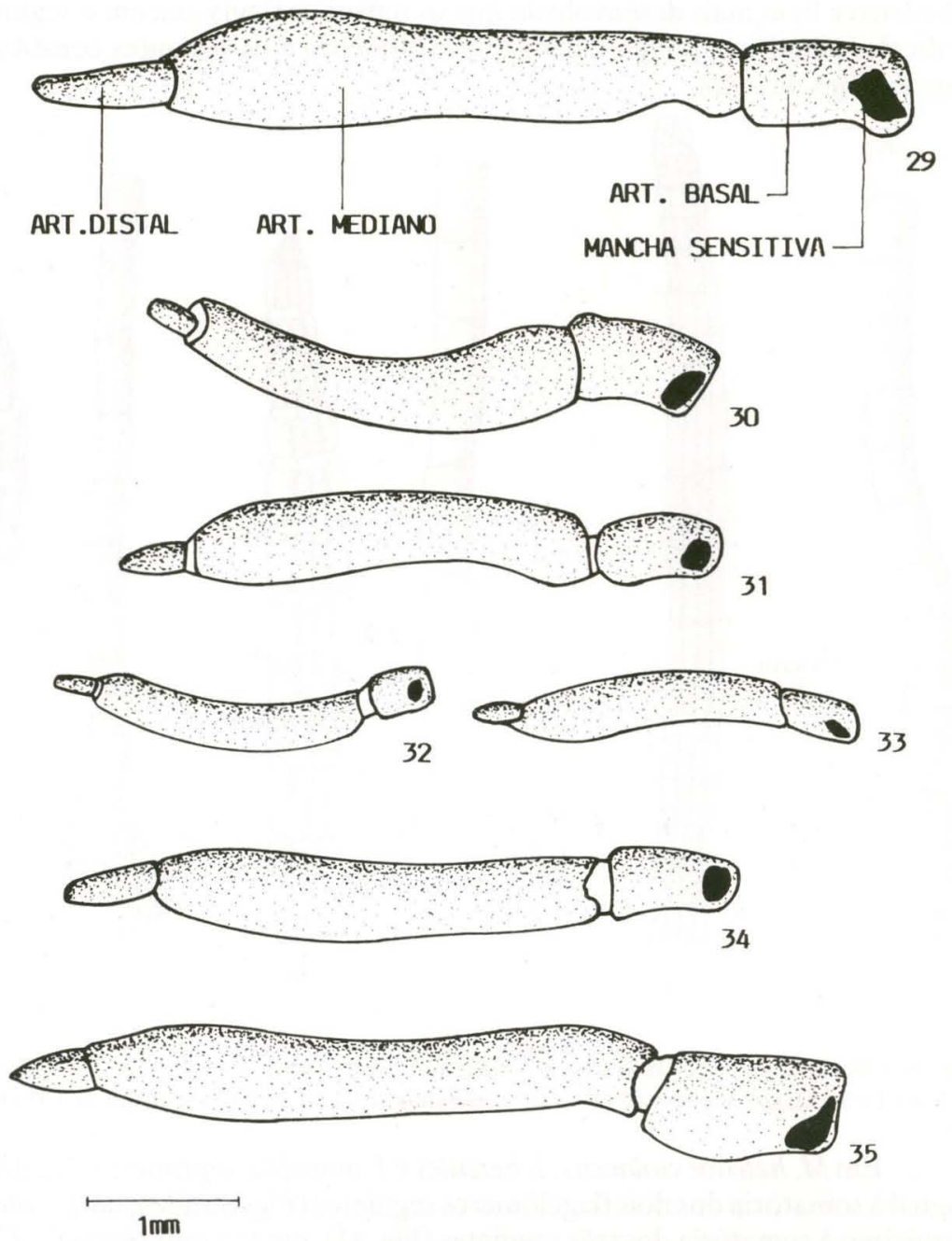

Figs 29 a 35. Palpo labial. (29) M.h. violaceus; (30) I. hercules; (31) I. anaxibia; (32) C.p. thamyris; (33) C. aega; (34) P. catenaria; (35) G.m. nestira.

aloja ao centro o lábio e aos lados as maxilas (Figs 22 a 28). Entre a fossa proboscidial e a pós-gena localiza-se a área hipostomal.

\section{APÊNDICES CEFÁLICOS}

As antenas localizadas nas margens dorsolaterais do frontoclípeo, são aproximadamente quatro vezes mais longas que a largura máxima da cabeça e não apresentam dimorfismo sexual. Uma variação no número de artículos é observada entre as espécies (42-58 artículos). O primeiro artículo, o escapo, é 
cilíndrico e bem mais desenvolvido que os outros, articula-se com o segundo, o pedicelo, cilíndrico, pouco menor que o primeiro, e os restantes constituem o flagelo (Figs 36 a 42).

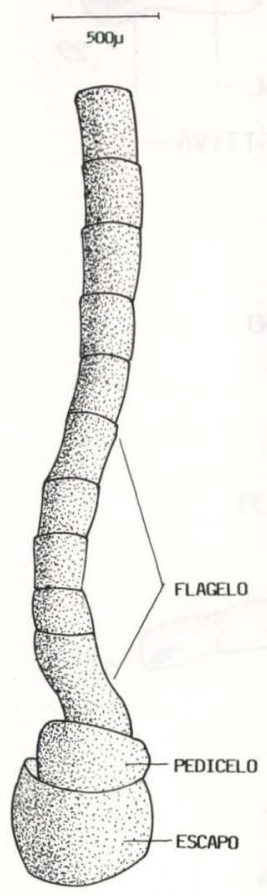

A

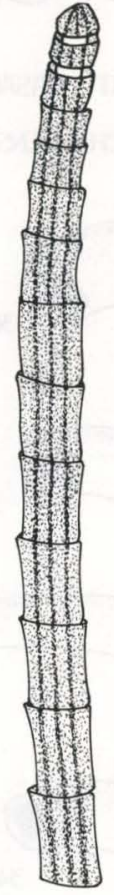

B

36

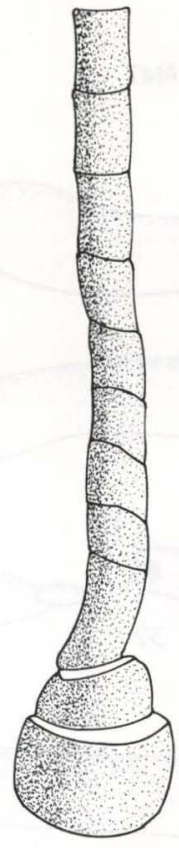

A

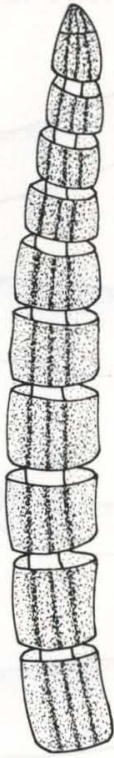

B

37

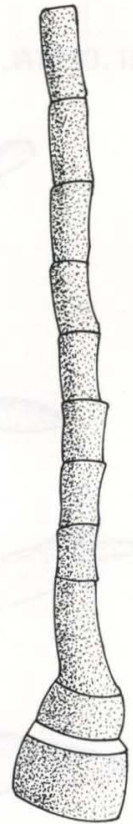

A

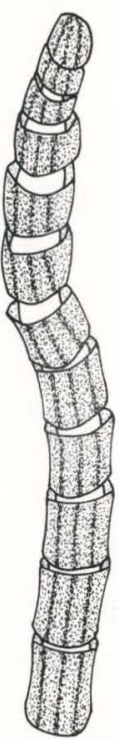

B

38

Figs 36 a 38. Antena. (36) M.h. violaceus, artículo 1 a 10 (A), artículo 43 a 56 (B); (37) I. hercules, artículo 1 a 9 (A), artículo 45 a 55 (B); (38) I. anaxibia, artículo 1 a 9 (A), artículo 34 a 45 (B).

Em M. helenor violaceus, I. hercules e I. anaxibia, o primeiro flagelômero é igual à somatória dos dois flagelômeros seguintes (Figs 36 a 38); em P. catenaria é próximo à somatória dos três seguintes (Fig. 41); em C. portis thamyris, C. aega e G. menelaus nestira é pouco maior que a somatória dos dois seguintes (Figs 39, 40 e 42). Quanto a forma, em M. helenor violaceus, I. hercules, I. anaxibia, C. portis thamyris e $P$. catenaria os flagelômeros pré-apicais são mais largos que os apicais (Figs 36 a 39 e 41) e $C$. aega e G. menelaus nestira os apicais são mais largos que os pré-apicais (Figs 40 e 42). Na margem interna das antenas, três carenas se estendem do primeiro flagelômero ao último, este de aspecto arredondado.

O labro, esclerito estreito e transversal, articula-se com o frontoclípeo através da sutura clipeolabral, possui um par de estruturas pilosas sobre as gáleas denominados pilíferos. No centro do labro está a epifaringe, pequeno lobo triangular e pouco esclerotinisado (Figs 1 a 7). 


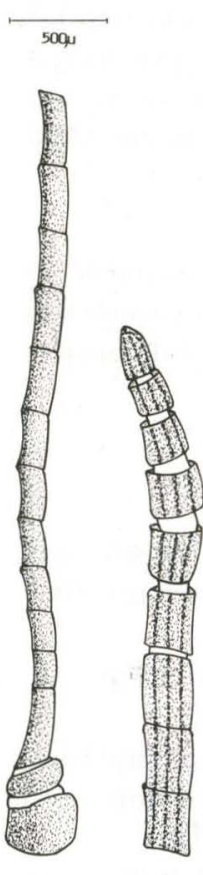

A
B

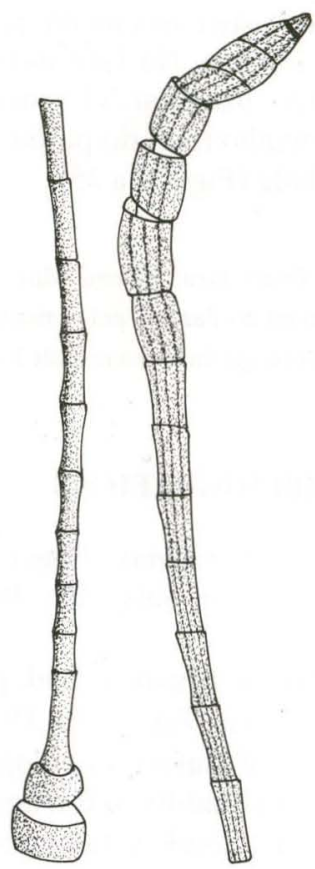

A
B

40

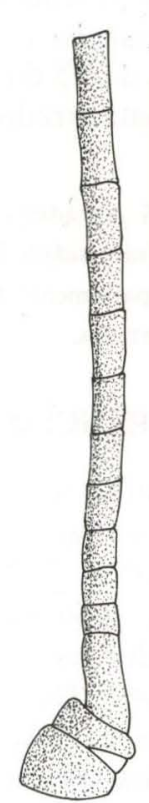

A

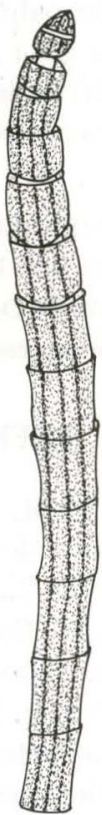

B

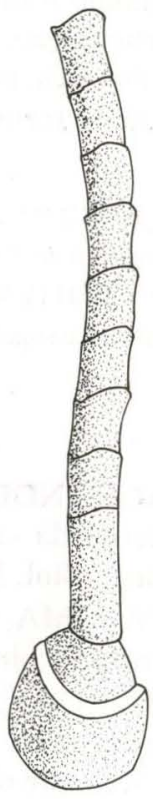

A

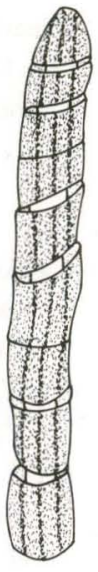

B

42

Figs 39-42. Antena. (39) C.p. thamyris, artículos 1 a 12 (A), artículo 32 a 40 (B); (40) C. aega, artículos 1 a 10 (A), artículos 20 a 34 (B); (41) P. catenaria, artículos 1 a 12 (A), artículos 36 a 49 (B); (42) G. m. nestira, artículos 1 a 9 (A), artículos 42 a 50 (B).

As maxilas ocupam a maior parte da fossa proboscidial. Os cardos, escleritos basais, pequenos e ovalados, em $M$. helenor violaceus, $I$. hercules e $C$. portis thamyris estão articulados anteriormente com os estípes (Figs 22, 23 e 25); enquanto que esta articulação não é observada em $I$. anaxibia, C. aega, $P$. catenaria e G. menelaus nestira (Figs 24, 26 a 28). Os estípes, escleritos desenvolvidos, com formas variadas, bem esclerotinisados e ladeados por membrana, estão projetados em direção às gáleas, circundando-as lateralmente e terminando abaixo dos pilíferos. Segundo CASAGRANDE (1979), são responsáveis pela sustentação das mesmas (Figs 22 a 28). Os palpos maxilares, aos lados do labro, são reduzidos (Figs 1 a 7). As gáleas ventralmente membranosas e dorsalmente pouco esclerotinisadas, convexas externamente, quando em repouso estão enroladas em espiral e encaixadas dentro da fossa proboscidial, quando distendidas são aproximadamente duas vezes e meia maior que a largura máxima da cabeça (Figs 1 a 7 e 22 a 28).

O lábio, esclerito triangular, ocupa o centro da fossa proboscidial, termina anteriormente em ponta entre as gáleas. Látero-posteriormente articula-se 
com os palpos labiais (Figs 15 a 28). Estes são bem desenvolvidos e triarticulados. $\mathrm{O}$ artículo basal é o mais robusto e levemente recurvado, tem 1/5 do tamanho total do palpo, articula-se ao lábio. $\mathrm{Na}$ face interna e proximal a esta articulação, há uma mancha sensitiva irregular. $\mathrm{O}$ artículo mediano é o maior dos três, pouco mais de $3 / 5$ do tamanho total do palpo. $\mathrm{O}$ artículo distal é o menor e termina em ponta arredondada (Figs 29 a 35).

AGRADECIMENTOS Agradeço à Prof Dra. Mirna Martins Casagrande do Departamento de Zoologia da Universidade Federal do Paraná, pela orientação e estímulo e ao Prof. Dr. Olaf H.H. Mielke do Departamento de Zoologia da Universidade Federal do Paraná pela co-orientação e sugestões apresentadas.

\section{REFERÊNCIAS BIBLIOGRÁFICAS}

CASAGRANDE, M.M. 1979. Sobre Caligo beltrao (Illiger). II. Morfologia externa da cabeça do adulto (Lepidoptera, Satyridae, Brassolinae). Rev. Brasil. Biol. 39 (1): 223-227.

COSTA LIMA, A.M. DA. 1950. Insetos do Brasil. Lepidoptera $2^{\text {a }}$ Parte, 6, Parte Morphidae. Rio de Janeiro, Esc. Nac. Agron., p. 356-361.

DOUBLEDAY, E. 1846-1852. The genera of diurnal Lepidoptera, comprising their generic characters, a notice of their habits and transformations, and a catalogue of the species of each genus. London, Longman, 534p.

EATON, J.L. 1971. Morphology of Head and Thorax of the adult Tobacco Hornworn, Manduca sexta (Lepidoptera: Sphingidae). 1. Skeleton and Muscles. Ann. Ent. Soc. Amer. 64 (2): 437-445.

EHRLICH, P.R. 1958a. The Integumental Anatomy of the Monarch Butterfly Danaus plexippus L. (Lepidoptera: Danaidae). Univ. Kansas Sci. Bull., Lawrence, 38 (18): 1315-1349.

. 1958b. The Comparative Morphology, Phylogeny and Higher Classification of the Butterflies (Lepidoptera: Papilionoidea). Univ. Kansas Sci. Bull., Lawrence, 39 (8): 305-369.

FRUHSTORFER, H. 1912-1924. Morphidae, p. 333-344 (1912), 345-356 (1913), pls. 66-71 (1924). In: A. SEITZ (ed.) Gross-Schmett. Erde. 5. Stuttgart, F. Lehman.

HAR VEY, D.J. 1991. Higher Classification of the Nymphalidae, p. 255-273. In: H.F. NIGHOUT (ed.) The development and evolution of butterfly wing pattern. Washington and London, Smithsonian Institution Press, 297p.

LE MOULT, E. \& P. RÉAL. 1962-1963. Les Morpho d'Amérique du Sud et Central. Historique-Morphologie-Systématique, 296p. (1962); vol. 2, Planches en couleurs et en noir, 92 pls (1963). Paris, Le Moult.

MADDEN, A.H. 1944. The external morphology of the adult of the Tobacco Hornworn (Lepidoptera, Sphingidae). Ann. Ent. Soc. Amer. 37 (2): 145-160. MATSUDA, R. 1965. Morphology and Evolution of the insect head. Mem. Amer. Ent. Inst. 4 (8): 212-221. 
NICULESCU, E. 1968. L'importance de l'exosquelette pour la Systematique des Lépidoptéres. Boll. Ass. Rom. Ent. 28 (2): 33-40.

1976. La valeur taxonomique des caractéres morphologiques chez les Lépidoptéres. Bull. Soc. Ent. Mulhouse: 1-14.

- 1978. Recherches de morphologie taxonomique dans l'ordre Lepidoptera. Bull. Soc. Ent. Mulhouse: 35-40.

REUTER, E. 1896. Ueber die Palpen der Rhopaloceren. Acta Soc. Scient. Fenn., Helsingfors, 22 (1): 1-577.

1898. On a New Classification of the Rhopalocera. Ent. Rec. \& J. Var., London, 10 (2): 25-98.

SBORDONI, V. \& S. FORESTIERO 1985. The world of butterflies, an illustrated encyclopedia. New York, Cies Crescent Books, 312p.

SHORT, J.R.T. 1951. Some aspects of the morphology of the insect head as seen in the Lepidoptera. Proc. R. Ent. Soc., London, 26: 77-88.

SNODGRASS, R.E. 1935. Principles of insect morphology. New York and London, MCGraw-Hill Book Company, 667p.

STAUDINGER, O. 1884-1888. p. 1-2 (1888); p. 3-38, pls. 1-30 (1884); p. 39-102, pls. $31-60$ (1885); p. 103-174, pls 61-80 (1886); p. 175-234, pls. $81-95$ (18870; p. 235-333, pls 96-100 (1888) In: O. STAUDINGER \& E. SCHATZ (ed.) Exotische Schmetterlinge. I. Theil, Exotische Tagfalter in systematischer Reihenfolge mit Berücksichtigung neuer Arten. Fuerth, Bayern.

Recebido em 01.III.1993; aceito em 13.VIII.1993. 